\title{
EL ROL DE LA INFRAESTRUCTURA TECNOLÓGICA EN RELACIÓN CON LA BRECHA DIGITAL Y LA ALFABETIZACIÓN DIGITAL EN 100 INSTITUCIONES EDUCATIVAS DE COLOMBIA
}

\author{
Luis Sánchez ${ }^{1}$ \\ Ana María Reyes ${ }^{2}$ \\ Diana Ortiz ${ }^{3}$ \\ Fredy Olarte ${ }^{4}$
}

\begin{abstract}
RESUMEN
La brecha digital es una problemática latente en América Latina, pese a las estrategias propuestas para contribuir a su reducción y que se enmarcan frecuentemente en políticas públicas tendientes a aumentar la infraestructura tecnológica y fortalecer la alfabetización digital. En el ámbito escolar, los procesos de alfabetización pueden tener un efecto sobre el desempeño académico de los estudiantes que puede medirse a través de pruebas estandarizadas. El objetivo de este estudio es caracterizar algunas condiciones de infraestructura tecnológica de instituciones educativas de diferentes zonas de Colombia, además de analizar la relación entre estas, los desarrollos tecnológicos y los resultados de la prueba estandarizada Saber 11. Para el estudio se consideraron 100 instituciones educativas públicas que participan en el proyecto Colegio 10 TIC. Se emplearon dos instrumentos para el diagnóstico de infraestructura eléctrica y tecnológica. Los datos fueron analizados mediante un estadístico no paramétrico de Kruskal-Wallis y un análisis de correspondencias múltiples (ACM). Aunque los resultados indican diferencias respecto del estado de la infraestructura tecnológica en distintas zonas del país, ninguna de ellas sobresale en desarrollos tecnológicos. Asimismo, no se observaron relaciones entre la infraestructura tecnológica de las instituciones educativas con los resultados de la prueba Saber 11 .
\end{abstract}

Palabras clave: alfabetización digital, brecha digital, infraestructura tecnológica, políticas públicas TIC.

Facultad de Ciencias Humanas, Universidad Nacional de Colombia, Bogotá, Colombia. Contacto: lasanchezru@unal.edu.co

2 Facultad de Ingeniería, Universidad Nacional de Colombia, Bogotá, Colombia. Contacto: amreyesp@unal.edu.co

3 Facultad de Ingeniería, Universidad Nacional de Colombia, Bogotá, Colombia. Contacto: dortiz@ciercentro.edu.co

4 Facultad de Ingeniería, Universidad Nacional de Colombia, Bogotá, Colombia. Contacto: faolarted@unal.edu.co 


\title{
THE ROLE OF TECHNOLOGICAL INFRASTRUCTURE IN RELATION TO THE DIGITAL DIVIDE AND DIGITAL LITERACY IN ONE HUNDRED EDUCATIONAL INSTITUTIONS IN COLOMBIA
}

\begin{abstract}
Despite efforts made to reduce the digital divide in Latin America, with strategies and policies focused on increasing technology infrastructure and strengthening digital literacy, a significant gap persists. In grade school, digital literacy processes can influence students' academic performance, which can be measured by standardized tests. This study presents an overview of and characterizes the technological infrastructure in public schools, from different Colombian regions, along with an analysis of the relationship between said infrastructure, technological developments and the overall results of the standardized test "Saber 11". The information was collected from one hundred public schools that participate in the project "Colegio 10 TIC," through two instruments designed to measure technological and electrical infrastructure. Data were analyzed using a nonparametric Kruskal-Wallis statistic and multiple correspondence analysis (MCA). Although the results show differences on the state of technological infrastructure in different regions across the country, not a single zone stands out, regarding technological developments. Furthermore, a relationship was not found between the condition of the school's technological infrastructure and the results reported on the "Saber 11" test. .
\end{abstract}

Keywords: digital divide, digital literacy, ICT public policies, technological infrastructure.

\section{Introducción}

Los desarrollos tecnológicos de las últimas décadas han marcado la diferencia en muchos contextos, desde el sector empresarial hasta las instituciones de educación. Es tanto así que en la actualidad se habla mucho de los beneficios de las Tecnologías de la Información y la Comunicación (TIC) en los entornos académicos, ya que contribuyen a la generación, transmisión y potenciación del conocimiento y el aprendizaje (Aguilar, 2012). No obstante, a pesar de que esta globalización de la tecnología está en boga, y ciertamente se ha hecho necesaria para la economía mundial y la misma cotidianidad de las personas, parece ser que a su vez ha contribuido a marcar más aún la diferencia entre países desarrollados y los que están en vías de desarrollo. Como lo destaca el Informe global sobre tecnología de la información 2016: innovando en la economía digital (Baller, Dutta \& Lanvin, 2016), no todos los países acceden a las bondades de las tecnologías o las usan de la misma manera. Gran parte de ellos, sobre 
todo en Latinoamérica, está en una etapa emergente o transicional del desarrollo digital (Baller et al., 2016). A esta diferencia de uso y acceso de las tecnologías entre grupos de población se le ha denominado brecha digital.

La brecha digital puede asociarse en cierta medida con un problema de política pública. Los lineamientos que los gobiernos definen - para que las necesidades alrededor de las nuevas tecnologías se satisfagan - no son siempre exitosos, incluso en todas las regiones de un país. En el caso específico de Colombia, estos lineamientos se pueden apreciar en el Plan Nacional de Desarrollo: 2010-2014 (Departamento de Planeación, 2011), en donde se destaca la importancia del fortalecimiento de las TIC para fomentar la inclusión, la superación de brechas (a nivel de infraestructura, disponibilidad de recursos y conectividad) y la apropiación de la tecnología a través del uso de contenidos. De esta manera, en el Plan se incorporaron iniciativas orientadas principalmente a la dotación masiva de recursos tecnológicos (en particular en instituciones educativas), la promoción de servicios TIC y el uso de internet, a fin de aportar en la disminución de la brecha digital y en el progreso para la innovación, productividad, competitividad y control social (Departamento Nacional de Planeación, 2011).

Aunque quedan claros los elementos que estructuran las políticas en torno al acceso y al uso de las tecnologías a nivel nacional, su implementación no parece tener el efecto esperado en la reducción de la brecha digital. Algunos autores argumentan que gobiernos como el de Colombia siguen una tendencia que se replica a nivel internacional, en donde las acciones para incorporar la tecnología en el sector educativo se enfocan principalmente en dotar y brindar infraestructura tecnológica, dejando en un segundo plano aspectos esenciales como el acompañamiento institucional para el uso de la infraestructura y la alfabetización digital de docentes y estudiantes (Vargas y Cruz, 2007). De esta manera, se evidencia cada vez más la necesidad de adelantar estudios que permitan tener un panorama general acerca de las iniciativas implementadas para integrar las TIC en el ámbito educativo, su efecto en la brecha de acceso y uso de las tecnologías y su posible relación con la calidad educativa. 
Respecto de esta última variable, es importante mencionar que existen diversos elementos que deberían considerarse para la medición y evaluación integral de la calidad educativa, los que tendrían que tomar en cuenta los múltiples factores que inciden en la misma (Krause, 2012). Algunos incluyen, por ejemplo, la pertinencia personal y social de lo que se enseña, la ética y profesionalismo de los maestros, el rol de los directivos, las alianzas entre escuelas u otros agentes educativos, el currículo, la cantidad y calidad de las didácticas, los incentivos socioeconómicos y culturales y, por supuesto, la cantidad, calidad y disponibilidad de los materiales educativos (Braslavsky, 2004). Aunque muchos de estos factores (relacionados con el rol de diferentes agentes educativos) son elementos fundamentales en la definición de la calidad educativa, estos no fueron contemplados en la presente investigación. Sí se consideró la relación entre uno de los indicadores de calidad educativa resultados en pruebas estandarizadas aplicadas a estudiantes-y los procesos de integración de infraestructura tecnológica en el contexto nacional, con una perspectiva de análisis enfocada principalmente hacia la alfabetización digital.

De esta manera, en este artículo se presentan inicialmente los resultados de un estudio en el que se analiza la infraestructura tecnológica de 100 instituciones educativas públicas (IE) de Colombia. Se enmarca en una iniciativa de política pública nacional denominada Plan Nacional Colegio 10 TIC, con el que se busca promover a algunas instituciones educativas a nivel nacional como pioneras en el uso de la tecnología y los recursos educativos digitales por parte de directivos, docentes y estudiantes, para contribuir de ese modo al mejoramiento de la calidad educativa en el país.

El objetivo principal del presente estudio es analizar las características de infraestructura tecnológica de las instituciones educativas que forman parte de la iniciativa Colegio 10 TIC, específicamente sus diferencias en aspectos como infraestructura, conectividad, desarrollos tecnológicos y espacios educativos tecnológicos, con el fin de tener un panorama acerca del acceso y el uso de la tecnología en el ámbito educativo a nivel nacional. Por otra parte, como un ejercicio inicial que permita obtener algunos indicios 
para profundizar en estudios posteriores en torno a la incidencia de la tecnología en la calidad educativa, se explorará si existe relación entre el estado de la infraestructura tecnológica de estos colegios y el rendimiento académico de los estudiantes en la prueba de Estado Saber 11. Esta prueba estandarizada, además de medir el desarrollo de las competencias de los estudiantes que están a punto de graduarse de educación media, puede considerarse como un indicador de la calidad educativa, ya que sirve para "monitorear la calidad de la educación de los establecimientos educativos con fundamento en los estándares básicos de competencias y los referentes de calidad emitidos por el Ministerio de Educación Nacional" (Ministerio de Educación Nacional de Colombia, 2016b,).

\section{Brecha digital}

La brecha digital se refiere a la diferenciación o separación producida entre individuos, grupos de individuos, instituciones, sociedades o países en el acceso y uso de las TIC. Puede ser definida en términos de la desigualdad de posibilidades que existen para acceder a la información, el conocimiento y la educación a través de la tecnología.

Esta situación, como bien podemos imaginarnos no es de presencia o ausencia, de estar o no estar, sino más bien de grado; es decir que la distancia, la brecha digital, de separación varía de un país a otro o de un colectivo hacia otro (Almenara, 2014, p. 15).

A la luz de las contribuciones de diferentes autores acerca del concepto de brecha digital, esta puede interpretarse desde tres perspectivas. En primer lugar, la brecha digital se asocia con las posibilidades de acceso a las TIC y se puede presentar debido a retrasos en la implementación de procesos de dotación tecnológica entre diferentes zonas y grupos sociales (Adriani \& Becchetti, 2003; Compaine, 2001 citado en Ferro, Helbig \& Gil-García, 2011). En segundo lugar, se plantea que la brecha digital no se sustenta exclusivamente en el acceso, sino que también considera el uso que los individuos o grupos poblacionales le dan a las TIC (GilGarcía, Helbig \& Ferro, 2006). Desde esta perspectiva también se consideran como elementos determinantes de la brecha digital la 
emergencia de factores endógenos y exógenos que están asociados a las características sociales y demográficas del país (Gil-García et al., 2006). Finalmente, la tercera perspectiva toma en cuenta la relación de la tecnología con aspectos más complejos como la raza, el género y la cultura para explicar la brecha digital (Castells, 2001; Civallero, 2006; Comisión Económica para América Latina y el Caribe, CEPAL, 2013; Kennedy, Wellman \& Klement, 2003; Warf, 2001 citados en Ferro et al., 2011). De acuerdo con esta última postura, no hay diferencias entre la forma en que la mayoría de las personas usa la tecnología, pero sí las hay en el propósito de uso de las tecnologías para las personas y las comunidades, lo cual suele estar asociado con configuraciones sociales e históricas específicas (Hines, Nelson \& Tu, 2001 citado en Ferro et al., 2011).

Considerando las diferentes perspectivas, el concepto de brecha digital podría desagregarse en dos elementos particulares: la brecha de acceso y la brecha de uso. En términos generales, se ha observado que las iniciativas gubernamentales asociadas a la reducción de la brecha digital se orientan predominantemente hacia la disminución de la brecha de acceso a través de la dotación masiva de dispositivos como computadores y tabletas, la ampliación en la cobertura de redes de internet y el incremento en las terminales para la conectividad. Las acciones que se han enmarcado en ampliar las oportunidades de acceso a las tecnologías para reducir este tipo de brecha se han potenciado, además, por el creciente uso de internet a través de diferentes tipos de dispositivos, incluyendo teléfonos celulares (Adhikari, Mathrani \& Parsons, 2015; Selwyn, 2006). Pese a los múltiples esfuerzos realizados para ampliar el acceso a la tecnología, estudios han revelado que estas iniciativas (frecuentemente limitadas a la dotación de infraestructura tecnológica) son insuficientes para fomentar la integración de las TIC al ámbito educativo y potenciar su uso pedagógico en el aula. Por ejemplo, en un estudio realizado en India, se propuso un escenario experimental para observar el uso que le daba un grupo de niños a los computadores que habían sido integrados a su entorno bajo un paradigma no invasivo de la educación, es decir, sin instrucción de algún docente (Warschauer, 2003). De acuerdo con los resultados de este estudio, los niños que 
usaron los computadores lo hicieron solo para jugar o para dibujar en ciertos programas (Warschauer, 2003).

Este tipo de experiencias deja entrever la importancia de ampliar el alcance de las iniciativas orientadas a la dotación masiva de infraestructura tecnológica, de manera que las políticas públicas devengan en acciones integrales que contemplen, además, procesos formativos y de acompañamiento para el uso de las nuevas tecnologías. Las estrategias que se formulan considerando este último tipo de acciones, se encuentran orientadas a reducir el otro componente de la brecha digital que parecía tener hasta el momento un papel secundario: la brecha digital de uso.

Como se mencionó previamente, la brecha digital de uso se relaciona con las diferencias que se presentan en el desarrollo de las habilidades necesarias para el manejo de las TIC. Este tipo de brecha parece estar íntimamente relacionada con los procesos de alfabetización digital que se realizan para que los diferentes grupos poblacionales puedan hacer uso de la tecnología de acuerdo con su contexto y necesidades particulares. De hecho, en algunas investigaciones se ha señalado a los procesos de alfabetización digital como uno de los factores clave para reducir la brecha digital (Adhikari et al., 2015; Reynolds \& Chiu, 2015; GértrudixBarrio, Gálvez de la Cuesta, SaidHung y Durán Medina, 2016). Estos procesos deben contemplar el acompañamiento docente al uso de nuevas tecnologías (Dorfsman, 2012), la formación en el manejo de las herramientas tecnológicas acorde con las condiciones socioeconómicas de la zona (Villarreal, 2012) y un enfoque de aprendizaje orientado a habilidades que se puedan transferir a otros dominios de la cotidianidad (Warschauer, 2003).

Considerando los elementos abordados hasta este momento, parece evidente que la reducción de la brecha digital (no solo de acceso, sino también de uso), se encuentra mediada por la introducción de políticas públicas que armonicen las iniciativas de dotación de infraestructura tecnológica con procesos de acompañamiento. Dichos procesos deben trascender la manipulación automatizada y sin propósito de los artefactos digitales, fomentando 
la formación de usuarios críticos de la tecnología y capacitados en su uso (Fourez, 1994).

\section{Políticas TIC en educación: contexto colombiano}

Las políticas públicas constituyen el mecanismo más común para que el gobierno de un país — a veces en conjunto con la poblaciónplasme los objetivos, decisiones y acciones para actuar sobre problemáticas específicas (Sistema de Información de Tendencias Educativas en América Latina, SITEAL, 2014). Las políticas TIC apuntan generalmente a la integración tecnológica en la educación formal, a fomentar la inclusión digital en poblaciones marginales y a reducir la brecha digital. Para la consecución de sus objetivos generales, estas políticas frecuentemente se proponen procesos de formación docente, desarrollo de contenidos y recursos digitales, alfabetización digital para docentes y estudiantes, entre otros (SITEAL, 2014).

En el contexto colombiano, desde la década de los ochenta se han propuesto políticas públicas relacionadas con las TIC y la educación. Estos referentes incluyen leyes y decretos a través de los cuales se ha desarrollado una estrategia nacional de ciencia, tecnología e innovación, como política económica y social del país. Dentro de los documentos que comprenden este marco jurídico, se destacan los Planes Nacionales Decenales de Educación y de TIC, los Planes Nacionales de Desarrollo y las Leyes No 1.341 y 1.286 (Galvis, Efrón y Rodríguez, 2014). De acuerdo con estos lineamientos de políticas públicas, en Colombia se ha estado implementando una serie de iniciativas encaminadas principalmente hacia la dotación de infraestructura informática y comunicacional (como Computadores para Educar, REDP, RENATA, Aula Innovadora, Colegio 10 TIC, etc.); el desarrollo de talento humano a través del uso de las TIC en educación (Pequeños Científicos, Escuela Virtual, Transformar la Práctica Docente y otros); la cualificación de la educación vía la innovación educativa con TIC (Congenia, Red Virtual de Tutores, etc.); y la producción y gestión de contenidos educativos de calidad en programas tales como la Eduteka, los Centros de Innovación Educativa y la Estrategia Nacional REDA (Galvis et al., 2014). 
120 EL ROL DE LA INFRAESTRUCTURA TECNOLÓGICA EN RELACIÓN CON LA BRECHA DIGITAL Y LA ALFABETIZACIÓN DIGITAL EN 100 INSTITUCIONES EDUCATIVAS DE COLOMBIA - L. Sánchez, A. Reyes, D. Ortiz y F. Olarte

El alcance de las políticas públicas y sus resultados se han visto afectados principalmente por dos factores: la obsolescencia tecnológica de los equipos entregados y la falta de continuidad en el desarrollo de las diversas iniciativas a través del tiempo (Galvis et al., 2014). Este último factor se debe principalmente a los cambios de gobernantes y a la carencia de recursos, lo que incide en las contrataciones de personal docente o encargado de capacitar y hacer seguimiento a los procesos de formación en alfabetización digital (Galvis et al., 2014). Estos factores, sumados a las falencias en la gestión de la infraestructura tecnológica al interior de algunas instituciones educativas, hacen que las pocas iniciativas que persiguen propósitos que van más allá de la dotación de infraestructura tecnológica se desarrollen parcialmente y no sean sostenibles en las diferentes instituciones en las que se realizan.

Cabe agregar que, como afirman Barón y Gómez (2012), los esfuerzos que hacen las organizaciones de la sociedad civil en comunión con organizaciones gubernamentales no han sido suficientes para dar un rumbo distinto a las políticas de Estado, las cuales siguen enfocándose primordialmente en el desarrollo de infraestructura y la instrumentación a nivel educativo (Barón y Gómez, 2012; SITEAL, 2014).

Tradicionalmente, el enfoque de las iniciativas que se proponen en el marco de las políticas TIC y el alcance que tienen en el contexto nacional parece sugerir que la disminución de la brecha digital de acceso (a través del aumento en la cobertura de las redes de internet y de la dotación masiva de recursos tecnológicos en instituciones públicas) se considera como el factor clave que, desde la perspectiva de las políticas TIC, puede contribuir a atenuar las situaciones de desigualdad que aquejan al país en general, incluyendo elementos relacionados con diferencias en la competitividad académica (Barón y Gómez, 2012). En los últimos años esta concepción ha cambiado gradualmente con el incremento en la formulación de proyectos orientados a la formación y acompañamiento de docentes y estudiantes en el uso pedagógico de la tecnología, iniciativas de las que se esperaría un efecto en la brecha digital de uso. Pese a los esfuerzos realizados para proponer iniciativas en esta línea de 
acción, aún se evidencia la necesidad de que las políticas públicas en TIC se enmarquen predominantemente desde una visión que involucre los rasgos múltiples que supone la brecha digital, como son: las características de las poblaciones, la calidad de las tecnologías y los usos asociados a ellas, los desarrollos y competencias que están fomentando las TIC y, sobre todo, los procesos de alfabetización digital (Durall, Gros, Maina, Johnson y Adams, 2012).

\section{Infraestructura tecnológica y alfabetización digital}

La alfabetización digital corresponde a un conjunto de estrategias cognitivas que los consumidores de la información digital utilizan al interactuar en ambientes tecnológicos (Jones-Kavalier \& Flannigan, 2008). Dicha alfabetización está mediada por el uso de una variedad de dispositivos -especialmente el computador - en los que los usuarios leen e interpretan la información, la reproducen y posteriormente pueden evaluarla y aplicarla en otros contextos (Jones-Kavalier \& Flannigan, 2008). A partir de lo anterior, surgen tres principios o niveles para comprender la alfabetización digital mencionados por Arrieta y Montes (2011): el primero responde al uso de herramientas digitales, programas y aplicaciones; el segundo obedece a la comprensión crítica de las TIC, o dicho de otra manera, a la habilidad de analizar y evaluar el contenido con el que se interactúa. Finalmente, el tercer nivel es aquel en donde el usuario es capaz de crear y compartir contenidos digitales haciendo uso de las herramientas tecnológicas. Aunque estos niveles suponen la disposición de infraestructura tecnológica como un factor fundamental para dar pie al acceso y uso de las TIC, los procesos de alfabetización digital van más allá de la dotación de dispositivos y la instrucción técnica para el manejo de nuevas tecnologías, sino que se enfocan en desarrollar en los usuarios las habilidades necesarias para fomentar escenarios de aprendizaje con tecnologías, manteniendo enfoques críticos, incluyentes y participativos.

Considerando esta mirada particular, los procesos de alfabetización digital constituyen una herramienta valiosa para apoyar estrategias de enseñanza-aprendizaje y fomentar el desarrollo de competencias en el ámbito educativo. De acuerdo con Eshet- 
Alkalai y Chajut (2009), en estudiantes de comunidades rurales la implementación de estrategias de alfabetización digital en el largo plazo ha tenido efectos significativos respecto del desempeño en tareas de reproducción de información, manipulación de imágenes, interacción con internet y en la creación de contenido crítico a partir de algo ya existente en la red. Por otro lado, otras investigaciones evidencian la importancia de la formación tecnológica como apuesta para desarrollar habilidades de lectura digital, escritura digital, uso de contenidos educativos y difusión ética de la información (Marzal, Parra y Colmenero, 2011). En otro sentido, otros autores destacan la importancia de esta alfabetización en el desarrollo de competencias ciudadanas (Gros y Contreras, 2006) y, sobre todo, como factor de inclusión social (Travieso y Planella, 2008). No obstante, cabe destacar que en la mayoría de estos estudios se enfatiza que los procesos de integración de las TIC en el ambiente escolar terminan enfocándose más en el desarrollo de habilidades instrumentales (Travieso y Planella, 2008) que en aquellas que tienen que ver con una postura crítica y creativa hacia el uso de la tecnología (EshetAlkalai \& Amichai-Hamburger, 2004).

En el contexto colombiano también se han desarrollado iniciativas para integrar las TIC en el ámbito educativo y se han elaborado estudios de investigación para evaluar su incidencia en escenarios de la educación básica y media. Computadores para Educar (CPE) constituye uno de los programas de mayor alcance a nivel nacional (con una cobertura del 96,2\% de los municipios), en el que se planteó como meta principal la disminución de la brecha digital mediante expansión del acceso, uso y aprovechamiento de las TIC en los espacios educativos públicos a nivel nacional (Orgales, Torres y Zúñiga, 2011). De acuerdo con algunos estudios realizados, este programa ha tenido un impacto positivo y significativo en la deserción académica; en los resultados de la prueba de Estado Saber 11; ha contribuido a aumentar la posibilidad de los estudiantes para acceder a la educación superior (Orgales, Torres \& Zúñiga, 2011; Ministerio de Educación y Ministerio TIC, s.f); y también ha incrementado el acceso y uso de computadores (Barrera-Osorio \& Linden, 2009). 
En contraposición con estos resultados, otro estudio de carácter cualitativo evidenció posturas encontradas en las perspectivas de los docentes y estudiantes respecto de los procesos de integración de las tecnologías en el ambiente educativo (Ministerio de Educación Nacional de Colombia y Ministerio de Tecnologías de la Información y las Comunicaciones de Colombia, s.f.). Por un lado, algunos de los participantes de este estudio resaltaban como elementos positivos asociados con el programa, las mejoras en la conectividad y disponibilidad de recursos tecnológicos, indicando además que su uso estaba contribuyendo a cerrar la brecha digital. Por otro lado, otros profesores y estudiantes destacaron falencias en la implementación del programa y en los procesos de acompañamiento pedagógico para el uso de nuevas tecnologías, mencionando la persistencia de una pedagogía tradicional enfocada en la transmisión, recepción y reproducción de la información y una visión de la tecnología restringida a ciertos espacios y áreas de aprendizaje, como la informática. Un último aspecto relevante de este estudio se relaciona con la percepción de que los procesos de capacitación en el uso de la tecnología se ven superados por el ritmo del cambio tecnológico, lo cual es consistente con el supuesto de que la infraestructura tecnológica en educación va a un nivel de desarrollo más rápido de lo que se puede incorporar en la enseñanza y el aprendizaje en las instituciones educativas (Moursund \& Bielefeldt, 1999).

En otro estudio local, realizado en instituciones educativas públicas y privadas de Barrancabermeja (Municipio de Santander), se resaltó la importancia de la infraestructura tecnológica en relación con el rendimiento de los estudiantes en la prueba de Estado Saber 11 (Centro de Estudios Regionales, CER, 2012). De acuerdo con los resultados de esta investigación, la infraestructura física es el factor que explica en mayor medida la calidad académica (CER, 2012). Para este estudio en particular, la infraestructura física está compuesta por espacios de esparcimiento, servicios públicos y por el área tecnológica (CER, 2012). Pese a identificar la infraestructura como elemento determinante en la calidad académica, los autores recomiendan que la inversión en ella debe ir acompañada de otras medidas, tales como incentivos a docentes y directivos y cambios en los modelos pedagógicos. Este último elemento también 
es reconocido como uno de los aspectos fundamentales asociados con la calidad educativa por parte de Gaviria y Barrientos (2001), en el marco de un estudio realizado en colegios públicos y privados de Bogotá.

En los diversos estudios que han abordado el tema, se evidencian tres elementos concretos. Primero, el interés gubernamental por enfocar las políticas públicas a la reducción de la brecha digital en el país, con el propósito de impactar la calidad educativa. Segundo, la existencia de una relación directa entre la infraestructura tecnológica y la alfabetización digital. En tercer y último lugar, se destacan posturas críticas basadas en estudios específicos que demuestran que si bien existen avances en el acceso, el uso de la infraestructura tecnológica no ha sido suficiente para impactar de manera directa la calidad académica en las instituciones educativas. Esto, claro está, sin considerar el impacto que contemplan otras variables que se escapan del alcance de esta investigación, como lo pueden ser aspectos culturales, pedagógicos, de roles directivos, entre otros.

En este artículo se presenta un análisis de los hallazgos sobre la infraestructura tecnológica de instituciones educativas públicas en Colombia, de las diferencias que existen en el contexto regional frente a este aspecto y la relación que tiene la infraestructura tecnológica con el desempeño académico de los estudiantes, medido a través de la prueba Saber 11. En síntesis, este estudio analiza la infraestructura tecnológica, la alfabetización digital y su impacto en la calidad educativa, medida a través de la prueba Saber 11, principal prueba estandarizada en el país.

\section{Método}

\subsection{Población}

El proceso de identificación de las características de la infraestructura tecnológica fue abordado por un grupo de instituciones educativas que forman parte de la iniciativa Colegio 10 TIC, la cual nace con el propósito de cumplir con uno de los objetivos del Gobierno nacional 
que es impulsar la educación en Colombia a través de las TIC en colegios públicos que son pioneros de la calidad educativa (Plan Nacional Colegio 10 TIC, Colombia Aprende, 2016). La primera etapa de implementación del proyecto consistió en el levantamiento de información diagnóstica en instituciones educativas a nivel nacional para temas de infraestructura tecnológica, avances pedagógicos en la apropiación de TIC y uso de recursos educativos digitales por parte de docentes y estudiantes.

El presente estudio trabajó sobre dicho levantamiento, en particular, sobre temas referidos a: infraestructura, conectividad, desarrollos tecnológicos y espacios educativos tecnológicos. La muestra correspondió a 100 colegios públicos de Colombia, los cuales fueron divididos en cinco zonas: centro, norte, occidente, oriente y sur. Estas zonas comprenden a los Departamentos de Cundinamarca, Bolívar, Valle del Cauca y Cauca, Meta y Antioquia, respectivamente. Todas las instituciones son de educación básica secundaria y solo 46 cuentan con formación básica primaria.

\subsection{Instrumentos y aplicación}

Para realizar el diagnóstico tecnológico de las instituciones educativas se diseñaron dos instrumentos: instrumento de diagnóstico eléctrico e instrumento de diagnóstico TIC. La validación de estos instrumentos fue realizada por un grupo de expertos en ingeniería y tecnologías en educación de la Universidad Nacional de Colombia. A través del instrumento de diagnóstico eléctrico se recogió información relacionada con la disponibilidad de equipos, características de la acometida eléctrica, cortes de energía, corrientes y voltajes del tablero principal; mientras que por medio del instrumento diagnóstico TIC, se obtuvo información asociada con la conectividad, los espacios tecnológicos, los recursos digitales y los desarrollos tecnológicos realizados en las instituciones educativas. La aplicación de los instrumentos fue ejecutada por un profesional vinculado con el proceso de caracterización del plan Colegio 10 TIC, quien obtuvo la información en el marco de visitas preliminares a las instituciones participantes entre los meses de octubre y noviembre de 2015. 
126 EL ROL DE LA INFRAESTRUCTURA TECNOLÓGICA EN RELACIÓN CON LA BRECHA DIGITAL Y LA ALFABETIZACIÓN DIGITAL EN 100 INSTITUCIONES EDUCATIVAS DE COLOMBIA - L. Sánchez, A. Reyes, D. Ortiz y F. Olarte

\subsection{Codificación y análisis de datos}

Para los propósitos de esta investigación se realizó una recodificación de las variables de los instrumentos de diagnóstico eléctrico y diagnóstico TIC, generando cuatro categorías que agrupan los aspectos más relevantes de la caracterización tecnológica:

- calidad del espacio educativo,

- condiciones eléctricas y de conectividad,

- recursos tecnológicos disponibles y

- desarrollo tecnológico de las instituciones educativas.

En la Tabla 1 se precisan los elementos asociados con estas categorías o variables.

Tabla 1

Categorías agrupadas de la caracterización tecnológica

\begin{tabular}{|c|c|c|c|}
\hline $\begin{array}{c}\text { Calidad de espacio } \\
\text { educativo }\end{array}$ & $\begin{array}{l}\text { Recursos } \\
\text { tecnológicos }\end{array}$ & $\begin{array}{l}\text { Desarrollo de } \\
\text { aplicaciones }\end{array}$ & $\begin{array}{l}\text { Condiciones } \\
\text { eléctricas y de } \\
\text { conectividad }\end{array}$ \\
\hline $\begin{array}{l}\text { Tipos de aula } \\
\text { disponibles en } \\
\text { las instituciones; } \\
\text { el estado de las } \\
\text { mismas; y la } \\
\text { seguridad para el } \\
\text { almacenamiento } \\
\text { de los dispositivos } \\
\text { tecnológicos y las } \\
\text { políticas de uso de } \\
\text { estos dentro de las } \\
\text { aulas. }\end{array}$ & $\begin{array}{l}\text { Recursos } \\
\text { tecnológicos } \\
\text { (hardware } \\
\text { y software) } \\
\text { disponibles en los } \\
\text { colegios como por } \\
\text { ejemplo: portátiles, } \\
\text { tabletas, periféricos, } \\
\text { kits educativos, etc. }\end{array}$ & $\begin{array}{l}\text { Uso de redes } \\
\text { sociales para } \\
\text { propósitos } \\
\text { institucionales } \\
\text { y académicos; } \\
\text { desarrollos en } \\
\text { robótica y de } \\
\text { aplicaciones; y uso } \\
\text { de aula virtual. }\end{array}$ & $\begin{array}{l}\text { Elementos de } \\
\text { infraestructura que } \\
\text { pueden incidir en } \\
\text { la conectividad } \\
\text { y el acceso a } \\
\text { la tecnología: } \\
\text { características de } \\
\text { la red eléctrica } \\
\text { (características de } \\
\text { las acometidas, } \\
\text { interrupciones } \\
\text { de energía, tipo } \\
\text { de cableado) y } \\
\text { de conectividad } \\
\text { (servidores, banda } \\
\text { ancha, etc.) }\end{array}$ \\
\hline
\end{tabular}

Fuente: Elaboración propia

A partir de esta categorización se realizó un análisis estadístico de las diferentes variables asociadas con la caracterización tecnológica de las instituciones educativas, considerando todas las zonas geográficas definidas para el presente estudio. Los análisis cuantitativos (realizados empleando el paquete estadístico SPSS versión 23 y el software estadístico R Studio) se desarrollaron para 
indagar en el tipo de relaciones que se producen entre las diferentes categorías propuestas y las zonas muestreadas, las cuales consideran los departamentos ya mencionados.

Por otra parte, con el fin de identificar agrupaciones de instituciones educativas que comparten características semejantes de infraestructura tecnológica, además de explorar las posibles relaciones entre esta variable y la calidad educativa (desde la perspectiva del desempeño de los estudiantes en pruebas estandarizadas), las categorías del diagnóstico tecnológico fueron analizadas junto con los resultados de la prueba de Estado Saber 11 del segundo semestre de 2015. La prueba Saber 11 es un examen estandarizado que aplica el Instituto Colombiano para la Evaluación de la Educación (ICFES) a estudiantes de undécimo grado, con el fin de medir las competencias básicas que deben desarrollar tras su paso por la escuela (Ministerio de Educación Nacional de Colombia, 2016b). El examen considera diferentes dimensiones de evaluación de competencias: Matemáticas, Lectura Crítica, Sociales y Ciudadanas, Ciencias Naturales e Inglés, Competencias Ciudadanas y Razonamiento Cuantitativo (Instituto Colombiano para la Evaluación de la Educación, ICFES, 2016a).

Para adelantar el análisis propuesto, se generó una nueva variable asociada con los resultados de la prueba Saber 11, la cual se calculó como el promedio de los puntajes correspondientes a las diferentes dimensiones del examen por institución educativa. Los resultados relacionados con las diferentes dimensiones del examen se obtuvieron a partir de la base de datos de la prueba Saber 11 2015-02, que se encuentra disponible en el repositorio de acceso público que tiene la página oficial del ICFES (ICFES, 2016b).

En cuanto a las metodologías de análisis empleadas en el presente estudio, se desarrolló un análisis estadístico no paramétrico para comparar el estado de infraestructura tecnológica en las diferentes zonas, mientras que para establecer la relación entre las variables del diagnóstico tecnológico y los resultados de la prueba Saber 11 se realizó un análisis de correspondencias múltiples (ACM). Dado que para este último se requiere que las variables sean de carácter nominal, fue necesario recodificar los resultados asociados a 
cada categoría (calidad del espacio educativo, condiciones eléctricas y de conectividad, recursos tecnológicos, desarrollo tecnológico y Pruebas Saber) en los siguientes niveles: alto, medio y bajo. El proceso de recodificación se realizó empleando como insumo la matriz TIC propuesta por Lugo y Kelly (2011), que constituye una aproximación inicial para la planificación de proyectos que involucran la integración de tecnología en el ambiente educativo a distintos niveles: inicial, intermedio y avanzado. Como resultado de este proceso de recodificación se definió una rúbrica que permite asignar un nivel (alto, medio o bajo) a cada categoría de acuerdo con las características de la infraestructura tecnológica propias de cada institución educativa. Para el caso de la variable prueba Saber 11, los niveles se definieron de acuerdo con los promedios asociados a las 100 instituciones educativas que forman parte del estudio, considerando que en general los resultados para esta agrupación de instituciones se encuentran muy por debajo del puntaje máximo de la prueba. En la Tabla 2 se presentan las características vinculadas con los distintos niveles de evaluación para cada una de las categorías de análisis.

Tabla 2

Niveles asociados a las categorías agrupadas para el ACM

\begin{tabular}{|c|c|c|c|}
\hline \multicolumn{4}{|c|}{ Nivel } \\
\hline Categorías & Alto & Medio & Bajo \\
\hline $\begin{array}{l}\text { Calidad de espacio } \\
\text { educativo }\end{array}$ & $\begin{array}{l}\text { Se dispone } \\
\text { de espacios } \\
\text { diferenciados para } \\
\text { la consecución } \\
\text { de las actividades } \\
\text { de tecnología } \\
\text { e informática, } \\
\text { los que están } \\
\text { además en buen } \\
\text { estado y permiten } \\
\text { implementar } \\
\text { políticas de } \\
\text { administración de } \\
\text { los espacios. }\end{array}$ & $\begin{array}{l}\text { Algunos de los } \\
\text { espacios disponibles } \\
\text { son de uso } \\
\text { compartido con } \\
\text { otras actividades } \\
\text { administrativas } \\
\text { y académicas; el } \\
\text { estado de las aulas } \\
\text { es regular y no } \\
\text { siempre cuentan } \\
\text { con políticas de uso } \\
\text { y administración. }\end{array}$ & $\begin{array}{l}\text { Por lo general lo } \\
\text { espacios poseen un } \\
\text { uso combinado; hay } \\
\text { una menor cantidad } \\
\text { de aulas para usos } \\
\text { específicos y estas } \\
\text { suelen estar en mal } \\
\text { estado y no cuentan } \\
\text { con políticas de uso } \\
\text { y administración. }\end{array}$ \\
\hline
\end{tabular}




\begin{tabular}{|c|c|c|c|}
\hline $\begin{array}{l}\text { Recursos } \\
\text { tecnológicos }\end{array}$ & $\begin{array}{l}\text { Además de } \\
\text { computadores de } \\
\text { escritorio, tanto } \\
\text { docentes como } \\
\text { estudiantes pueden } \\
\text { disponer de } \\
\text { otros dispositivos } \\
\text { específicos tales } \\
\text { como: cámaras } \\
\text { digitales, pizarras } \\
\text { electrónicas, } \\
\text { portátiles, tabletas y } \\
\text { kits educativos. }\end{array}$ & $\begin{array}{l}\text { Se dispone de } \\
\text { computadores de } \\
\text { escritorio y, en } \\
\text { menor medida, } \\
\text { de dispositivos } \\
\text { específicos: cámaras } \\
\text { digitales, pizarras } \\
\text { electrónicas, } \\
\text { portátiles, tabletas } \\
\text { y kits educativos. } \\
\text { Estos poseen un uso } \\
\text { diferenciado según } \\
\text { alumnos o docentes. }\end{array}$ & $\begin{array}{l}\text { Los recursos son } \\
\text { limitados, se } \\
\text { cuenta con algunos } \\
\text { dispositivos como } \\
\text { computadores } \\
\text { de escritorio y } \\
\text { portátiles, pero } \\
\text { en cantidades } \\
\text { reducidas y no } \\
\text { siempre funcionales. }\end{array}$ \\
\hline $\begin{array}{l}\text { Desarrollos } \\
\text { tecnológicos }\end{array}$ & $\begin{array}{l}\text { Las instituciones } \\
\text { disponen de página } \\
\text { web con recursos } \\
\text { digitales y cuentan } \\
\text { con redes sociales } \\
\text { tanto de uso } \\
\text { institucional como } \\
\text { académico. Hacen } \\
\text { uso de kits de } \\
\text { robótica y procuran } \\
\text { escenarios para } \\
\text { el desarrollo de } \\
\text { aplicaciones. }\end{array}$ & $\begin{array}{l}\text { Las instituciones } \\
\text { cuentan con página } \\
\text { web (por lo general } \\
\text { desactualizada) y } \\
\text { con redes sociales } \\
\text { institucionales. } \\
\text { Algunas veces hacen } \\
\text { uso de herramientas } \\
\text { especializadas } \\
\text { tales como kits } \\
\text { de robótica y, } \\
\text { eventualmente, } \\
\text { propician escenarios } \\
\text { para el desarrollo de } \\
\text { aplicaciones. }\end{array}$ & $\begin{array}{l}\text { Las instituciones no } \\
\text { cuentan con una } \\
\text { página web, por lo } \\
\text { general no hacen } \\
\text { uso de redes sociales } \\
\text { para propósitos } \\
\text { institucionales ni } \\
\text { académicos. No } \\
\text { hay un enfoque } \\
\text { en el desarrollo } \\
\text { de aplicaciones } \\
\text { o proyectos de } \\
\text { robótica. }\end{array}$ \\
\hline $\begin{array}{l}\text { Condiciones } \\
\text { eléctricas y de } \\
\text { conectividad }\end{array}$ & $\begin{array}{l}\text { La institución } \\
\text { cuenta con buenas } \\
\text { condiciones de } \\
\text { intranet e internet. } \\
\text { El equipamiento de } \\
\text { las aulas es óptimo. } \\
\text { Se presentan } \\
\text { cortes de energía } \\
\text { esporádicamente. }\end{array}$ & $\begin{array}{l}\text { La conectividad } \\
\text { es restringida, } \\
\text { pero funcional y } \\
\text { estable. El tipo } \\
\text { de equipamiento } \\
\text { para conectividad } \\
\text { es moderado y sus } \\
\text { condiciones físicas } \\
\text { son regulares. Hay } \\
\text { menos de tres cortes } \\
\text { de energía al día. }\end{array}$ & $\begin{array}{l}\text { La conectividad de } \\
\text { internet es débil e } \\
\text { insuficiente. Las } \\
\text { condiciones del } \\
\text { equipamiento no } \\
\text { son las mejores } \\
\text { y son apenas } \\
\text { suficientes. Los } \\
\text { cortes de energía } \\
\text { pueden llegar a ser } \\
\text { cuatro o más al día. }\end{array}$ \\
\hline $\begin{array}{l}\text { Prueba Saber } 11 \\
(2015-02)^{*}\end{array}$ & $\begin{array}{l}\text { Los puntajes } \\
\text { promedio oscilan } \\
\text { entre } 0 \text { y } 47,6 \text {. }\end{array}$ & $\begin{array}{l}\text { Los puntajes } \\
\text { promedio están } \\
\text { entre } 47,7 \text { y } 49,5\end{array}$ & $\begin{array}{l}\text { Los puntajes } \\
\text { promedio están } \\
\text { entre } 49,6 \text { y } 55,9 \text {. }\end{array}$ \\
\hline
\end{tabular}

Nota: * sobre las 100 instituciones muestreadas.

Fuente: Elaboración propia. 
130 EL ROL DE LA INFRAESTRUCTURA TECNOLÓGICA EN RELACIÓN CON LA BRECHA DIGITAL Y LA ALFABETIZACIÓN DIGITAL EN 100 INSTITUCIONES EDUCATIVAS DE COLOMBIA - L. Sánchez, A. Reyes, D. Ortiz y F. Olarte

\section{Resultados}

\subsection{Análisis estadístico no paramétrico de Kruskal-Wallis}

Para comparar las características tecnológicas entre las zonas de análisis propuestas en el presente estudio, se realizó el análisis estadístico no paramétrico de Kruskal-Wallis, el cual permite la comparación de tres o más grupos independientes (las zonas en este caso) para determinar cuáles de las diferencias entre ellos no son producto del azar. Mediante esta prueba se logró establecer que hay diferencias significativas entre las zonas respecto de las variables de calidad de espacio educativo $(=38,34, p<0,05)$, recursos tecnológicos $(=41,87, p<0,05)$, condiciones eléctricas y de conectividad $(=12,91, p<0,05)$ y también en cuanto al promedio de la prueba Saber 11 2015-02 $(=13,22, p<0,05)$. Esto indica que las diferencias que se presentan entre las zonas respecto de las categorías tecnológicas enunciadas y de los resultados de la prueba Saber 11 están relacionadas con el hecho de que una institución educativa pertenece a una zona en particular, y no al azar. Por otra parte, no se identificaron diferencias significativas en cuanto a los desarrollos tecnológicos $(=7,90, p>0,05)$ de las zonas evaluadas, como se puede apreciar en la Tabla 3. Este resultado puede presentarse debido a que la mayoría de las instituciones educativas reportaron niveles muy bajos en el desarrollo de proyectos de innovación tecnológica, actividades relacionadas con el uso de herramientas especializadas (como kits de robótica) y desarrollo de aplicaciones.

Tabla 3

Análisis no-paramétrico: Kruskal-Wallis para las categorías de caracterización tecnológica

\begin{tabular}{lccccc}
\hline \multicolumn{5}{c}{ Estadísticos de contraste ${ }^{\mathrm{a}, \mathrm{b}}$} \\
\hline & $\begin{array}{c}\text { Calidad } \\
\text { espacio } \\
\text { educativo }\end{array}$ & $\begin{array}{c}\text { Recursos } \\
\text { tecnológicos }\end{array}$ & $\begin{array}{c}\text { Desarrollos } \\
\text { tecnológicos }\end{array}$ & $\begin{array}{c}\text { Promedio } \\
\text { Saber 11 } \\
\text { 2015-02 }\end{array}$ & $\begin{array}{c}\text { Conectividad } \\
\text { general }\end{array}$ \\
\hline Chi-cuadrado & 38,341 & 41,877 & 7,903 & 13,226 & 12,918 \\
Gl & 4 & 4 & 4 & 4 & 4 \\
Sig. asintót. & 0,000 & 0,000 & 0,095 & 0,010 & 0,012 \\
\hline
\end{tabular}

a. Prueba de Kruskal-Wallis

b. Variable de agrupación: Zona

Fuente: Elaboración propia 
La Figura 1 permite visualizar las diferencias entre las zonas que agrupan a las 100 instituciones educativas en relación con los puntajes de las categorías de infraestructura tecnológica y de las prueba Saber 11. Estos puntajes fueron transformados en valores Z, los cuales se expresan en unidades de desviación estándar y se aprecian con una dirección y grado respecto de la media. De acuerdo con los valores presentados en la Figura 1, se puede observar que la zona sur y occidente tienen las mejores características tecnológicas en cuanto a la calidad del espacio educativo y a las condiciones eléctricas y de conectividad. Asimismo, las zonas occidente y oriente destacan entre las demás en la categoría relacionada con recursos tecnológicos. Por otra parte, se puede apreciar que la zona norte se encuentra muy rezagada respecto de las demás en las diferentes categorías de infraestructura tecnológica.

Por otro lado, se hace evidente que en la categoría de desarrollos tecnológicos no se aprecian grandes diferencias entre las zonas de análisis. No obstante, se destacan algunas instituciones de las zonas occidente, centro y oriente, que han comenzado con acciones orientadas a fomentar desarrollos tecnológicos. Esto es consistente con los resultados del análisis de Kruskal-Wallis en el que, pese a que se estableció que no se presentaban diferencias significativas entre la categoría de desarrollos tecnológicos y las zonas, el puntaje asociado con este tipo de análisis (chi cuadrado: 7,903) deja ver que hay instituciones que han procurado desarrollos en este sentido.

Finalmente, respecto de los puntajes de la prueba Saber 11 2015-02, se observa que aunque existen diferencias significativas entre las zonas (de acuerdo con el análisis de Kruskal-Wallis), los puntajes promedio de las instituciones educativas pertenecientes a las diferentes departamentos superan marginalmente el puntaje promedio de 50, el cual fue establecido por el ICFES para esta prueba a nivel nacional. Pese a que este indicador alcanza, en términos generales, un nivel bajo en las instituciones que forman parte de este estudio, la zona sur presenta los mejores resultados en esta prueba estandarizada. 


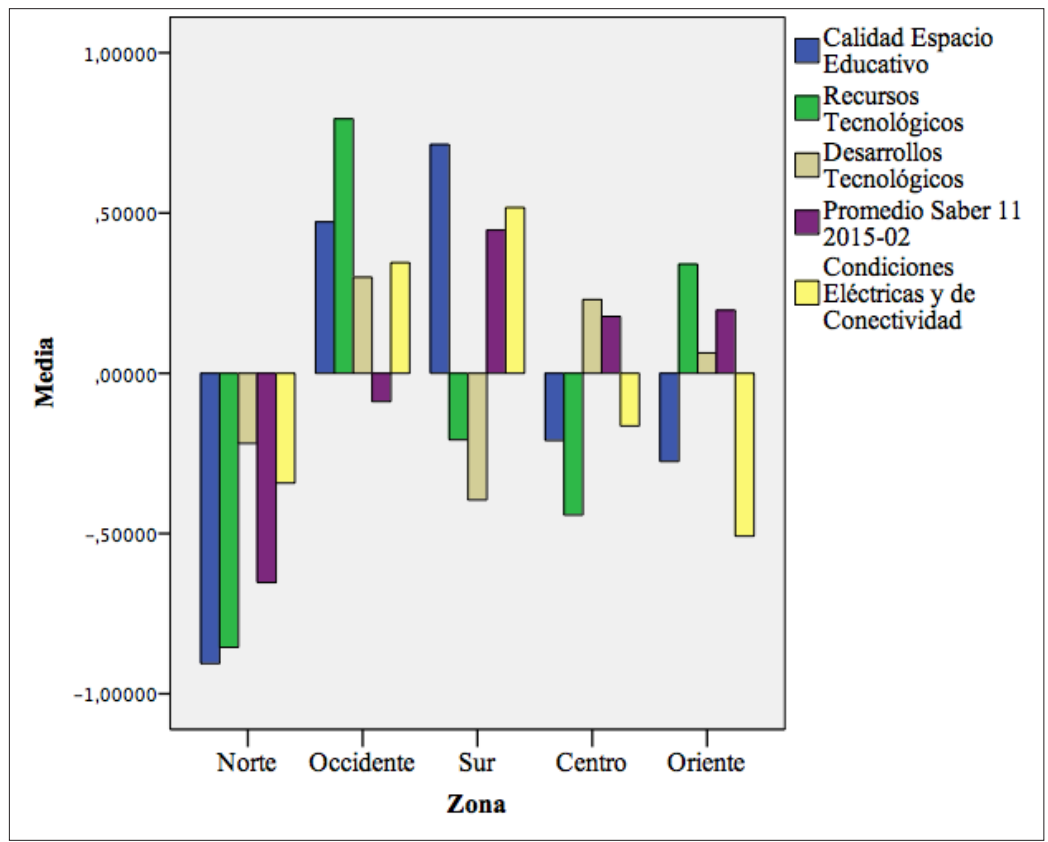

Figura 1. Puntajes Z de las categorías de caracterización tecnológica y la prueba Saber 11 con respecto a las cinco zonas de muestreo de instituciones educativas.

Fuente: Elaboración propia.

\subsection{Análisis de correspondencias múltiples (ACM)}

El análisis de correspondencias múltiples (ACM) es una técnica descriptiva cuyo objetivo es agrupar datos en un número reducido de dimensiones o factores, situando en un plano bidimensional tanto a las categorías (infraestructura tecnológica y prueba Saber 11) como a los sujetos involucrados en el análisis (instituciones educativas) (Pérez, 2013). De esta manera, para este estudio en particular, el ACM permite visualizar simultáneamente todas las variables (categorías de infraestructura tecnológica y prueba Saber 11) y sus relaciones con las instituciones educativas. Como parte de la metodología del ACM, se desarrolla inicialmente un análisis factorial que permite definir la cantidad de factores alrededor de los que se realiza el ACM. Estos constituyen los ejes explicativos que resumen la información del conjunto de variables, estableciendo la cantidad de aglomeraciones de sujetos (instituciones educativas) que, en alguna medida, presentan 
similitudes en las diferentes categorías de análisis. En la Tabla 4 se encuentran los cinco primeros factores obtenidos en el análisis factorial de correspondencias múltiples. En este caso, cada valor propio se relaciona con la varianza explicada por cada uno de los factores, la cual va aumentando de un factor a otro. Para este estudio se usaron y analizaron solo los cinco primeros factores, teniendo en cuenta que estos explican el 78,2\% de la varianza total y que al considerar los tres factores restantes se observó información redundante.

Tabla 4

Cinco primeros factores en el ACM

\begin{tabular}{cccc}
\hline Número & Valor propio & Porcentaje & Porcentaje acumulado \\
\hline 1 & 0,495 & 24,79 & 24,79 \\
\hline 2 & 0,329 & 16,49 & 41,28 \\
\hline 3 & 0,278 & 13,94 & 55,23 \\
\hline 4 & 0,254 & 12,74 & 67,97 \\
\hline 5 & 0,205 & 10,27 & 78,24 \\
\hline
\end{tabular}

Fuente: Elaboración propia

En la Figura 2 se presenta el plano bidimensional de análisis generado para los cinco primeros factores del ACM. En concordancia con la cantidad de factores usados para el análisis, se puede observar que se forman cinco clases dentro de las que es posible agrupar las instituciones educativas, de acuerdo con los niveles asociados a las categorías de infraestructura tecnológica. 


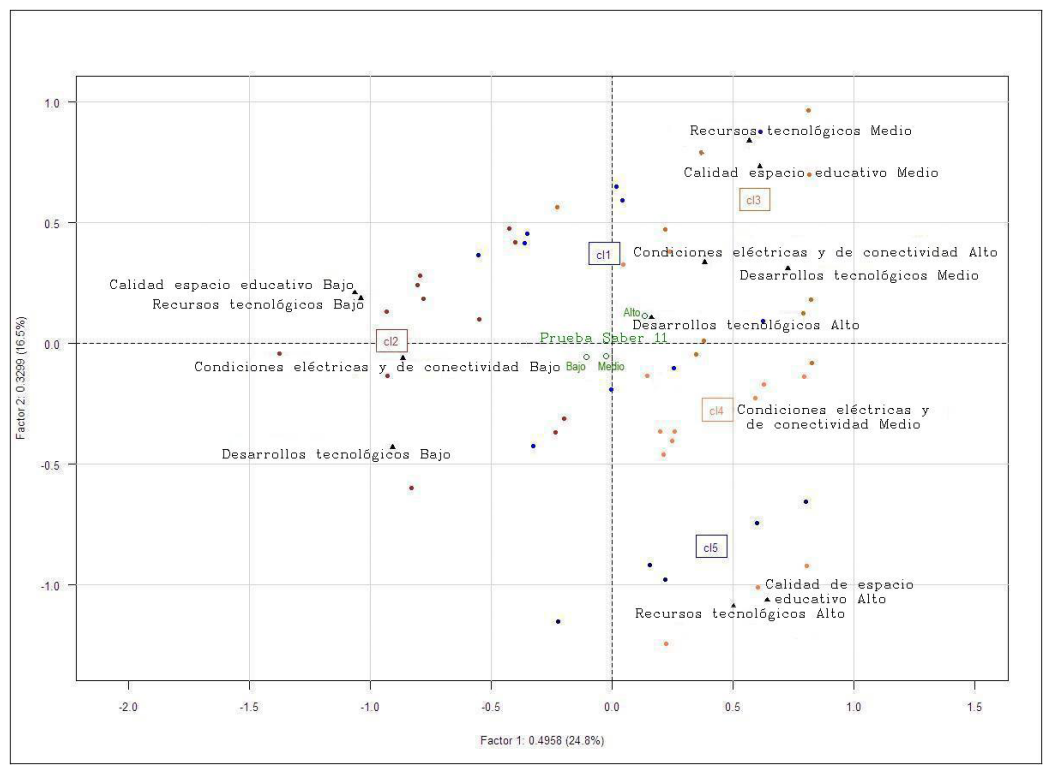

Figura 2. Plano factorial del ACM para las cinco clases; en donde cl1, cl2, cl3, cl4 y cl5 corresponden a las cinco clases analizadas.

Fuente: Elaboración propia.

A continuación se realiza la descripción de las características de infraestructura tecnológica para las diferentes clases que emergieron tras el ACM, considerando los niveles propuestos para las categorías de análisis (ver Tabla 2).

- Clase 1. Esta primera clase agrupa el 15\% del total de las instituciones educativas (IE) que forma parte del estudio. El $34 \%$ de las instituciones educativas que pertenecen a esta clase cuenta con condiciones eléctricas y de conectividad alta, lo que quiere decir que dispone de conexiones a internet eficientes, rápidas y de buena calidad en la mayoría de los casos. Además son instituciones que no suelen tener cortes de energía. En cuanto a los desarrollos tecnológicos, el 28\% de las IE de esta clase presenta un nivel alto en cuanto a esta categoría. Esta clase agrupa las IE que sobresalen respecto de las demás en la implementación de actividades de desarrollo de aplicaciones, uso de redes sociales para actividades institucionales, académicas y proyectos de robótica y/o electrónica. 
- Clase 2. Esta segunda clase reúne el 29\% de todas las instituciones de la muestra, las cuales se agrupan con niveles bajos en cuanto a la calidad del espacio educativo (31\% de las IE de esta clase), condiciones eléctricas y de conectividad (22\% de las IE de esta clase), recursos tecnológicos (25\% de las IE de esta clase) y desarrollos tecnológicos (21\% de las IE de esta clase). En otras palabras, la mayoría de las instituciones que pertenecen a esta clase no cuenta con espacios diferenciados para el desarrollo de actividades académicas y administrativas, la conectividad a internet es de baja calidad e insuficiente y suele haber entre cuatro o más cortes de energía al día. Adicionalmente, son instituciones con baja disponibilidad y calidad de recursos tecnológicos, lo cual también puede incidir en los niveles bajos reportados en la categoría de "desarrollos tecnológicos".

- Clase 3. En esta clase se ubica el 18\% del total de IE, cuyas características en términos de desarrollos tecnológicos (30\% de las IE de esta clase), recursos tecnológicos (30\% de las IE de esta clase) y calidad del espacio educativo (22\% de las IE de esta clase) corresponden a un nivel intermedio. Específicamente, estas instituciones cuentan con algunos espacios de uso compartido y el estado en el que se encuentran dichas aulas es regular; disponen del equipamiento necesario para poder trabajar en ellas, pero no siempre es suficiente ni está en las mejores condiciones. De la misma manera, una proporción considerable de las IE de esta clase se caracteriza por contar con página web y redes sociales institucionales (frecuentemente desactualizadas) $y$, eventualmente, hacen uso de herramientas especializadas tales como kits de robótica.

- Clase 4. Esta cuarta clase incluye el 27\% de las instituciones educativas de la iniciativa Colegio 10 TIC, las cuales cuentan con un nivel alto de recursos tecnológicos (17\% de las IE de esta clase), niveles intermedios en términos de la calidad del espacio educativo (18\% de las IE de esta clase) y condiciones eléctricas y de conectividad (32\% de las IE de esta clase). Se trata entonces de instituciones en las que las aulas son de uso compartido (administrativo y académico), la conectividad a internet es restringida (pero funcional y estable) y en las que, además de los 
136 EL ROL DE LA INFRAESTRUCTURA TECNOLÓGICA EN RELACIÓN CON LA BRECHA DIGITAL Y LA ALFABETIZACIÓN DIGITAL EN 100 INSTITUCIONES EDUCATIVAS DE COLOMBIA - L. Sánchez, A. Reyes, D. Ortiz y F. Olarte

recursos tecnológicos básicos, se disponen de otros dispositivos específicos para ser utilizados por parte de estudiantes y docentes.

- Clase 5. En esta clase (integrada por el $11 \%$ de las IE) se encuentran las instituciones que cuentan con niveles altos en términos de calidad de espacio educativo (30\% de las IE de esta clase), recursos tecnológicos (32\% de las IE de esta clase) y condiciones eléctricas y de conectividad (25\% de las IE de esta clase). Estas instituciones, además de disponer de recursos tecnológicos específicos para uso de docentes y estudiantes (característica común con las IE de la clase 4), cuentan con espacios educativos diferenciados, en buen estado y con políticas de uso y administración de tecnologías. A esto se le suman buenas condiciones de conectividad a intranet e internet y estabilidad en el suministro de energía eléctrica.

Finalmente, en la Figura 2 también se puede observar la distribución espacial de los niveles asociados con la categoría de la prueba Saber 11. Para el presente análisis, los resultados de la prueba Saber 11 fueron considerados como una variable de proyección. Esto implica que esta variable se presenta como un elemento ilustrativo sobre el plano factorial para dar cuenta con mayor precisión de su relación con las otras variables y/o categorías. En este caso, se observa una baja contribución de la variable "prueba Saber 11" en las clases emergentes del ACM, lo cual se debe en parte a la ubicación centralizada de los distintos niveles asociados con esta categoría en particular. La clase 3 es la única agrupación donde se identifica la presencia de la categoría de prueba Saber 11, en la que el 18\% de las IE que pertenece a esta clase presenta niveles altos en los puntajes de la prueba. La baja contribución de la variable en las clases refleja una relación débil entre las características tecnológicas de las instituciones educativas y el nivel de desempeño de los estudiantes de grado once.

Estos resultados sugieren que el poco impacto que tiene la infraestructura tecnológica en la calidad educativa de algunas instituciones, considerando que la prueba Saber 11 es uno de los máximos referentes en este aspecto. De acuerdo con la literatura, este fenómeno puede darse debido a que se presentan algunas falencias 
por parte de los docentes (asociadas con su arraigo hacia los modelos pedagógicos tradicionales) en la adopción e implementación de TIC en el aula (Ministerio de Educación Nacional de Colombia y Ministerio de Tecnologías de la Información y las Comunicaciones de Colombia, s.f.) y a la implementación de modelos restringidos de dotación tecnológica por parte de las entidades encargadas de fomentar la integración TIC en el ámbito educativo (Travieso y Planella, 2008).

\section{Discusión}

La literatura en torno a la alfabetización digital enfatiza en la importancia de los procesos de formación que promueven en los estudiantes habilidades que se salgan del esquema tradicional instrumental (Travieso y Planella, 2008), situando a la infraestructura tecnológica en un papel complementario, pero no principal. Los resultados de esta investigación dan cuenta de falencias en este sentido, sugiriendo que la integración de las TIC en las instituciones educativas de la muestra se ha limitado frecuentemente a la introducción de infraestructura tecnológica, dejando de lado el desarrollo de procesos integrales de alfabetización digital. Esto se evidencia en los bajos niveles de desarrollos tecnológicos de las IE y en la débil relación entre las categorías de infraestructura tecnológica y los resultados en pruebas estandarizadas (prueba Saber 11).

Aunque podría considerarse que las acciones orientadas hacia la ampliación de la infraestructura tecnológica en IE dan cuenta de un interés desde las políticas públicas por reducir la brecha digital de acceso (Barón y Gómez, 2012), los hallazgos de esta investigación muestran que persisten diferencias respecto de las características de infraestructura tecnológica en las diferentes zonas del país y sus respectivos departamentos (exceptuando en la categoría relacionada con desarrollos tecnológicos). De esta manera, se evidencia una brecha de acceso no solo en términos de equipamiento tecnológico y conectividad a internet, sino también en relación con el estado de los recursos tecnológicos y la calidad de los espacios disponibles para usarlos en el ambiente educativo. Las instituciones educativas de la región occidente presentan las mejores condiciones de infraestructura 
138 EL ROL DE LA INFRAESTRUCTURA TECNOLÓGICA EN RELACIÓN CON LA BRECHA DIGITAL Y LA ALFABETIZACIÓN DIGITAL EN 100 INSTITUCIONES EDUCATIVAS DE COLOMBIA - L. Sánchez, A. Reyes, D. Ortiz y F. Olarte

tecnológica, mientras que las de la zona norte muestran falencias en todas las categorías.

Otro de los hallazgos del análisis comparativo entre zonas mostró que no existen diferencias significativas respecto de la variable de "desarrollos tecnológicos", debido principalmente a los niveles bajos reportados para esta en la mayoría de instituciones educativas. Considerando que ella puede interpretarse como un indicador asociado al uso de la infraestructura tecnológica en las IE, podría inferirse que las iniciativas ejecutadas hasta el momento en las instituciones de la muestra no han tenido una mayor incidencia en la alfabetización digital y en aportar de manera considerable en la integración pedagógica de las TIC al aula. Además, estos resultados plantean nuevas interrogantes relacionadas con el rol de los diferentes agentes educativos (docentes, directivos docentes, padres de familia y estudiantes) en los procesos de uso y apropiación de las nuevas tecnologías que llegan a estas instituciones educativas. No obstante, es importante recordar que la información analizada en el presente estudio se obtuvo durante la primera etapa diagnóstica de la iniciativa Colegio 10 TIC, por lo que sería valioso evaluar el impacto de este programa una vez se concluya la etapa de implementación del mismo, integrando variables de análisis adicionales que permitan dar cuenta del nivel de apropiación de las TIC por parte de diferentes agentes educativos.

Por otro lado, el análisis de correspondencias múltiples deja ver la forma cómo se aglomeran las instituciones educativas de acuerdo con las categorías de infraestructura tecnológica contempladas. Los resultados de este análisis muestran que al menos un 74\% de las instituciones presenta niveles bajos e intermedios en las condiciones eléctricas y de conectividad, calidad del espacio educativo, desarrollos tecnológicos y recursos tecnológicos. De acuerdo con esto, aún faltan esfuerzos por mejorar las condiciones tecnológicas en el sistema educativo nacional, particularmente, en las zonas norte y oriente. Asimismo, y en consonancia con las afirmaciones de la Organización para la Cooperación y el Desarrollo Económicos (Organisation for Economic Co-operation and Development, OECD, 2015), este análisis permitió dilucidar que en los resultados de la prueba estandarizada 
de calidad educativa Saber 11 no se está evidenciando el impacto de una alfabetización digital que trascienda la formación instrumental y la dotación de infraestructura tecnológica a instituciones educativas. En general, la débil relación entre la prueba Saber 11 y las características tecnológicas de las IE puede asociarse con la falta de escenarios auténticos de integración de las TIC en el aula, en los que los diferentes agentes educativos lideren procesos de apropiación educativa (Spreen \& Knapczyk, 2017), a partir de las capacidades adquiridas en programas orientados al desarrollo de competencias para la integración de tecnologías. Asimismo, la relación entre estas variables (prueba Saber 11 y características tecnológicas) también puede dar cuenta de un bajo nivel de madurez en los procesos de integración de las TIC en estas instituciones educativas (BarreraOsorio \& Linden, 2009). No obstante, para validar estas afirmaciones sería necesario integrar a este análisis otras variables que exceden el alcance del presente trabajo.

De igual manera, estos resultados también podrían interpretarse desde el análisis de otras variables no consideradas en este estudio, como por ejemplo, aspectos sociales, culturales, las metodologías empleadas por los docentes para incluir las tecnologías en la enseñanza y los modelos de enseñanza-aprendizaje adoptados por cada una de las instituciones educativas.

En conclusión, a pesar de la existencia de diversas políticas públicas decantadas a través de iniciativas que tienen como propósito cerrar la brecha digital, se siguen perpetuando acciones que se limitan a una alfabetización digital cernida a los usos básicos de las tecnologías. Con ello, se están restringiendo las posibilidades de formar usuarios críticos, empoderados y creativos, capaces de trasferir sus habilidades digitales a otros entornos no solo académicos sino cotidianos (Warschauer, 2003) y de evidenciarlas en pruebas estandarizadas nacionales.

Al respecto, esta investigación apoya las perspectivas de otros estudios que resaltan la importancia de prácticas pedagógicas extendidas (Benavides y Pedró, 2007), ancladas al uso de TIC y consecuentes con una alfabetización digital participativa (Eshet- 
140 EL ROL DE LA INFRAESTRUCTURA TECNOLÓGICA EN RELACIÓN CON LA BRECHA DIGITAL Y LA ALFABETIZACIÓN DIGITAL EN 100 INSTITUCIONES EDUCATIVAS DE COLOMBIA - L. Sánchez, A. Reyes, D. Ortiz y F. Olarte

Alkalai \& Chajut, 2009), democrática (Gros y Contreras, 2006) e incluyente (Travieso y Planella, 2008), que favorezcan tanto a estudiantes como a docentes.

Aunque este estudio presenta los resultados de una muestra reducida de instituciones educativas de Colombia, constituye una perspectiva valiosa que desde una mirada del contexto nacional (considerando IE de distintas regiones del país), busca aportar al análisis de la incidencia que tienen los procesos de integración de TIC en el ambiente educativo a nivel nacional y regional.

Los resultados de la investigación reafirman la necesidad de formular e implementar políticas públicas que articulen acciones para la dotación, ampliación y mejora de la infraestructura tecnológica, con estrategias que garanticen su uso apropiado, diferenciado y proclive al desarrollo de una alfabetización digital armonizada con las necesidades y contextos de docentes y estudiantes; sin dejar de lado, claro está, la responsabilidad de los agentes educativos en la construcción de conocimiento con los recursos tecnológicos disponibles. De esta manera, se podrá considerar una verdadera integración pedagógica de las TIC en el ambiente educativo, que repercuta en el ámbito social y académico.

\section{Referencias}

Adhikari. J., Mathrani., A., \& Parsons. D. (2015). Bring your own devices classroom: Issues of digital divides in teaching and learning contexts. Trabajo presentado en Australasian Conference on Information Systems, University of South Australia, Adelaida, Australia.

Aguilar, M. (2012). Aprendizaje y tecnologías de información y comunicación: hacia nuevos escenarios educativos. Revista Latinoamericana de Ciencias Sociales, Niñez y Juventud, 10(2), 801-811.

Almenara, J. C. (2014). Reflexiones sobre la brecha digital y la educación. Revista del Hospital Interzonal General de Agudos (HIGA) Eva Perón, 2(4), 14-26.

Arrieta, A. C. y Montes, V. D. (2011). Alfabetización digital: uso de las TIC's más allá de una formación instrumental y una buena infraestructura. Revista Colombiana de Ciencia Animal 3(1), 180-197. Recuperado de https://dialnet.unirioja.es/servlet/articulo?codigo $=3691443$ 
Baller, S., Dutta, S., \& Lanvin, B. (Eds.) (2016). The global information technology report. Innovation in the digital economy. Recuperado de http://www3.weforum.org/docs/GITR2016/WEF_GITR_Full_Report. pdf

Barón, L. F. y Gómez, R. (2012). De la infraestructura a la apropiación social: panorama sobre las políticas de las tecnologías de información y comunicación (TIC) en Colombia. Signo y Pensamiento, 31(61), 38-55.

Barrera-Osorio, F. \& Linden, L. L. (2009). The use and misuse of computers in education: Evidence from a randomized experiment in Colombia (Working Paper 4836). World Bank Policy Research.

Benavides, F. y Pedró, F. (2007). Políticas educativas sobre nuevas tecnologías en los países iberoamericanos. Revista Iberoamericana de Educación, 45, 19-69.

Braslavsky, C. (2004). Diez factores para una educación de calidad para todos en el siglo XXI. Documento Básico. Presentado en la XIX Semana Monográfica de la Educación "Educación de calidad para todos": Iniciativas Iberoamericanas. Santillana, Madrid.

Castells, M. (2001). Conclusión: la sociedad en red. En La era de la información: economía, sociedad y cultura, (Vol. 1, 3ra ed.). México: Siglo veintiuno editores.

Centro de Estudios Regionales, CER. (2012). Relación existente entre la infraestructura y los resultados de las pruebas SABER 11: de las instituciones educativas de Barrancabermeja, Santander. Recuperado de http://cer.org. co/Documentos/resumenejecutivol1.pdf

Civallero, E. (noviembre, 2006). La brecha digital y su amenaza en Latinoamérica. Trabajo presentado en el IX Congreso Nacional de Bibliotecarios. Asociación Ecuatoriana de Bibliotecarios, Riobamba, Ecuador.

Comisión Económica para América Latina y el Caribe, CEPAL (2013). Análisis de la integración de la perspectiva de género en las agendas y políticas digitales de Latinoamérica y el Caribe. Recuperado de https://goo.gl/uBkhVp.

Departamento Nacional de Planeación (2011). Plan Nacional de Desarrollo. Bogotá. Recuperado de https://goo.gl/MAOLPD

Dorfsman, M. I. (2012). La profesión docente en contextos de cambio: el docente global en la sociedad de la información. Recuperado de https://www.um.es/ead/reddusc/6/marcelo_dusc6.pdf 
142 EL ROL DE LA INFRAESTRUCTURA TECNOLÓGICA EN RELACIÓN CON LA BRECHA DIGITAL Y LA ALFABETIZACIÓN DIGITAL EN 100 INSTITUCIONES EDUCATIVAS DE COLOMBIA - L. Sánchez, A. Reyes, D. Ortiz y F. Olarte

Durall, E., Gros, B., Maina, M., Johnson, L., y Adams, S. (2012). Perspectivas tecnológicas: educación superior en Iberoamérica 2012-2017. Austin, Texas: The New Media Consortium.

Eshet-Alkali, Y. \& Amichai-Hamburger, Y. (2004). Experiments in digital literacy. Cyberpsychology \& Behavior, 4(7), 421-429. https://doi.org/10.1089/cpb.2004.7.421

Eshet-Alkalai, Y. \& Chajut, E. (2009). Changes over time in digital literacy. Cyberpsychology \& Behavior, 6(12), 713-715. https://doi.org/10.1089/cpb.2008.0264

Ferro, E., Helbig, N. C., \& Gil-García, J. R. (2011). The role of IT literacy in defining digital divide policy needs. Government Information Quarterly, 1(28), 3-10. https://doi.org/10.1016/j.giq.2010.05.007

Fourez, G. (1994). Alfabetización científica y tecnológica. Acerca de las finalidades de la enseñanza de las ciencias. Buenos Aires: Colihue.

Galvis, A., Efrón, L., y Rodríguez, G. (2014). Las políticas TIC en los sistemas educativos de América Latina: caso Colombia. Recuperado de https://www.unicef.org/argentina/spanish/Colombia_WEB.pdf

Gaviria, A. y Barrientos, J. (2001). Características del plantel y calidad de la educación en Bogotá. Coyuntura Social, 25, 81-9 8.

GértrudixBarrio, F., Gálvez de la Cuesta, M., SaidHung, E. y DuránMedina, F. (2016). Alfabetización digital, competencias mediáticas y Open Data: Digital Literacy, Media Literacy and Open Data. RELATEC, 2(15), 113121.

https://doi.org/10.17398/1695-288X.15.2.113

Gil-García, J. R., Helbig, N., \& Ferro, E. (2006). Is it only about Internet access? An empirical test of a multi-dimensional digital divide. Lecture Notes in Computer Science, 139-149.

https://doi.org/10.1007/11823100_13

Gros, B. y Contreras, D. (2006). La alfabetización digital y el desarrollo de competencias ciudadanas. Revista Iberoamericana de Educación, 42, 103-125.

Instituto Colombiano para la Evaluación de la Educación, ICFES. (2016a). Información de la prueba Saber 11. Recuperado de http://www.icfes.gov.co/instituciones-educativas-y-secretarias/ saber-11/informacion-de-la-prueba-saber1 1

Instituto Colombiano para la Evaluación de la Educación, ICFES (2016b). Resultados Nacionales prueba Saber 11. Recuperado de 
http://www.icfes.gov.co/instituciones-educativas-y-secretarias/ saber-11/resultados-agregados-saber-11/resultados-agregados-2015-2

Jones-Kavalier, B. R. \& Flannigan, S. L. (2008). Connecting the digital dots: Literacy of the 21st century. Teacher Librarian, 3(35), 13-16.

Krause, K. L. (2012). Addressing the wicked problem of quality in higher education: Theoretical approaches and implications. Higher Education Research \& Development, 31(3), 285-297.

https://doi.org/10.1080/07294360.2011.634381

Lugo, M. T. y Kelly, V. (2011). La matriz TIC. Una herramienta para planificar las Tecnologías de la Información y Comunicación en las instituciones educativas. Buenos Aires: IIPE- Unesco.

Marzal, M. A., Parra, P., y Colmenero, M. J. (2011). La medición de impacto y evaluación de programas de alfabetización en información para bibliotecas escolares. Revista Española de Documentación Científica, 34(2), 190-211.

https://doi.org/10.3989/redc.2011.2.780

Ministerio de Educación Nacional de Colombia (2016b). Pruebas Saber. Recuperado de http://www.mineducacion.gov.co/1759/w3-article-244735.html

Ministerio de Educación Nacional de Colombia y Ministerio de Tecnologías de la Información y las Comunicaciones de Colombia (s.f.). Evaluación de impacto y de la sostenibilidad de Computadores para Educar en la calidad de la educación en las sedes educativas. Recuperado de http://www.computadoresparaeducar.gov.co/sites/default/files/ inlinefiles/Articulo_impacto_Computadores_para_Educar_Colombia. pdf

Moursund, D. \& Bielefeldt, T. (1999). Will new teachers be prepared to teach in a digital age? A national survey on information technology in teacher education. Recuperado de https://www.researchgate.net/publication/248450194_Will_New_ Teachers_Be_Prepared_To_Teach_in_a_Digital_Age

Organisation for Economic Co-operation and Development, OECD. (2015). Students, computers and learning: Making the connection, PISA. Recuperado de http://www.mecd.gob.es/dctm/inee/internacional/pisa-2012-studentscomputers.pdf?documentId=0901e72b81e9cc75

Orgales, C. R., Torres, F. S., y Zúñiga, J. M. (2011). Impacto del Programa Computadores para Educar en la deserción estudiantil, el logro escolar y el ingreso a la educación superior. Recuperado de https://ideas.repec.org/p/col/000089/008744.html 
144 EL ROL DE LA INFRAESTRUCTURA TECNOLÓGICA EN RELACIÓN CON LA BRECHA DIGITAL Y LA ALFABETIZACIÓN DIGITAL EN 100 INSTITUCIONES EDUCATIVAS DE COLOMBIA - L. Sánchez, A. Reyes, D. Ortiz y F. Olarte

Pérez, J. L. (2013). La estadística: una orquesta hecha instrumento. Tema 27: análisis de correspondencias. Recuperado de https://estadisticaorquestainstrumento.wordpress.com/2013/07/06/ tema-27-analisis-de-correspondencias/

Reynolds, R. \& Chiu, M. M. (2015). Reducing digital divide effects through student engagement in coordinated game design, online resource use, and social computing activities in school. Journal of the Association for Information Science and Technology, 67(8), 1822-1835. https://doi.org/10.1002/asi.23504

Selwyn, N. (2006). Digital division or digital decision? A study of non-users and low-users of computers. Poetics, 4-5(34), 273-292. https://doi.org/10.1016/j.poetic.2006.05.003

Sistema de Información de Tendencias Educativas en América Latina, SITEAL. (2014). Informe sobre tendencias sociales y educativas en América Latina 2014. Buenos Aires: Unesco.

Spreen, C. \& Knapczyk, J. J. (2017). Measuring quality beyond test scores: The impact of regional context on curriculum implementation (in Northern Uganda). Forum for International Research in Education, FIRE, 1(4). Recuperado de http://preserve.lehigh.edu/fire/vol4/issl/1

Travieso, J. L. y Planella, J. (2008). La alfabetización digital como factor de inclusión social: una mirada crítica. Recuperado de http://www.uoc.edu/uocpapers/6/dt/esp/travieso_planella.pdf

Vargas, G. G. y Cruz, J. C. (2007). Políticas tecnológicas en un escenario de gestión del conocimiento en educación. Revista Iberoamericana de Educación, 45, 71-88.

Villarreal, N. (2012). Proyecto Aulas Fundación Telefónica del Programa Proniño Panamá: una experiencia de educación inclusiva con el uso de las TIC. En G. Sunkel y D. Trucco (Eds.), Las tecnologías digitales frente a los desafíos de una educación inclusiva en América Latina: algunos casos de buenas prácticas (pp. 211-231). Santiago de Chile: CEPAL.

Warschauer, M. (2003). Demystifying the digital divide. Scientific American, 289(2), 42-47.

https://doi.org/10.1038/scientificamerican0803-42

Recibido: 26/09/2016

Aceptado: 07/08/2017 